\title{
Using the Update of Conditional BFGS in Constrained Optimization
}

\section{Abbas Y. Al-Bayati}

profabbasalbayati@yahoo.com
Received on: 12/03/2003
Ban Ahmed Mitras

dr.banah.mitras@gmail.com

College of Computer Sciences and Mathematics

University of Mosul, Iraq

\section{ABSTRACT}

Accepted on: 26/01/2004

In this paper, we have used one of the preconditioned conjugate gradient algorithm with the Quasi - Newton approximation; namely the BFGS preconditioned algorithm which was suggested by (AL-Bayati and Aref, 2001). In this paper we have suggested a new algorithm for constrained optimization with robust numerical results, for solving constrained optimization problems.

Keyword: Constrained Optimization, QN-Condition, BFGS Update.

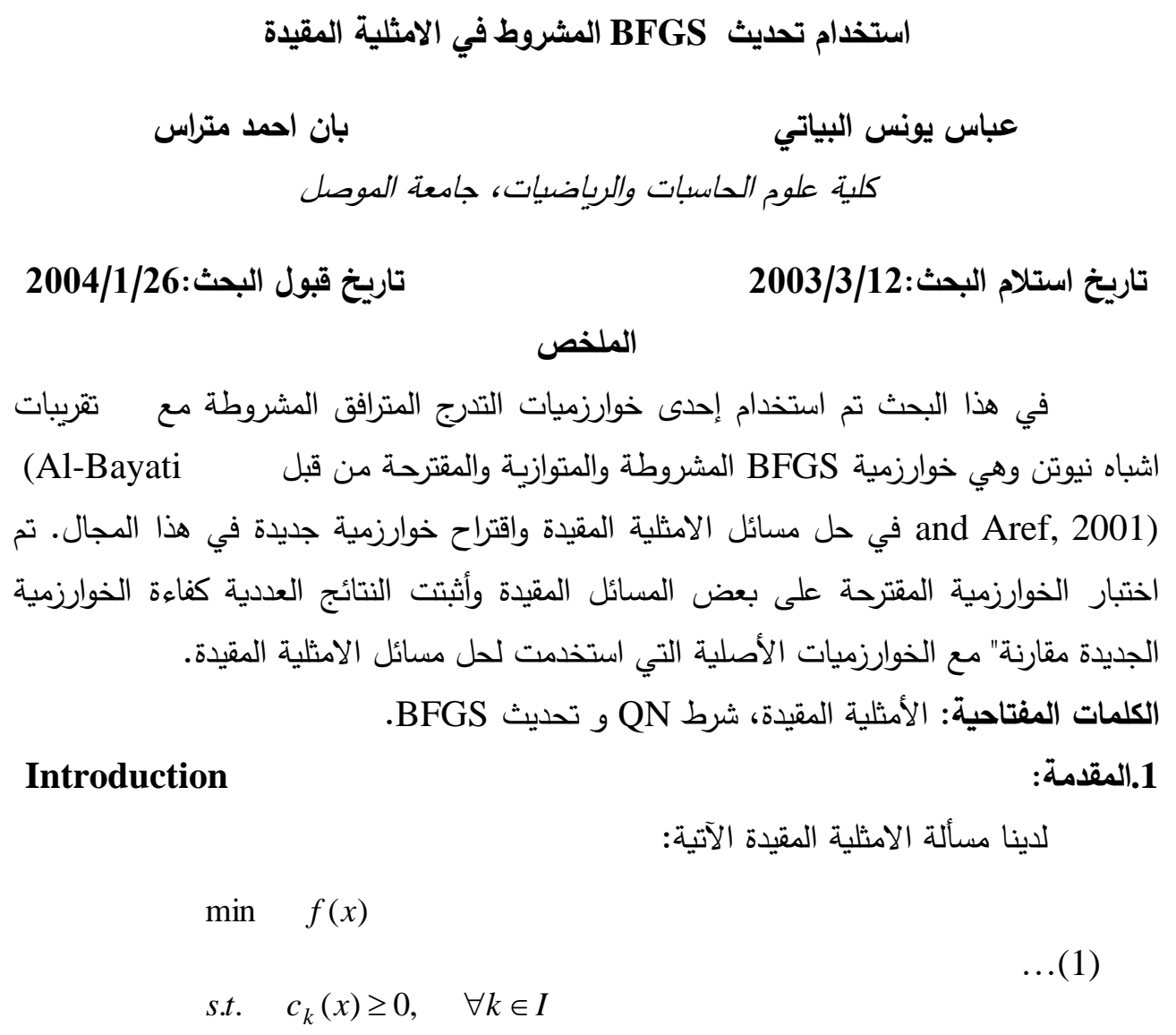




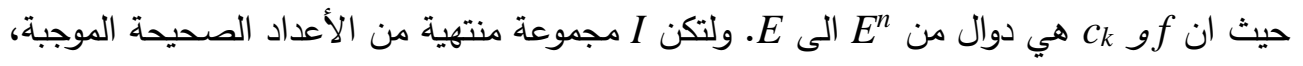

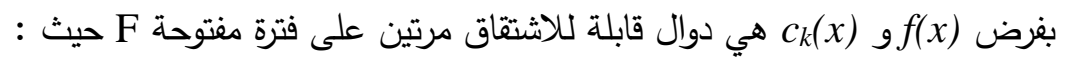

$$
F=\left\{x \in E^{n}: c_{k}(x) \geq 0, \quad \forall k \in I\right\}
$$

$$
\begin{aligned}
& \theta(x, r)=f(x)+r B(x) \\
& \text { من الممكن إيجاد حل لمسألة مقيدة عامة باستخدام دالة هدف محوة } 1(3)
\end{aligned}
$$

حيث أن r تعرف على انها معلمة سيطرة (3) (control parameter) والحد الثاني (B) هو دالة Barrier

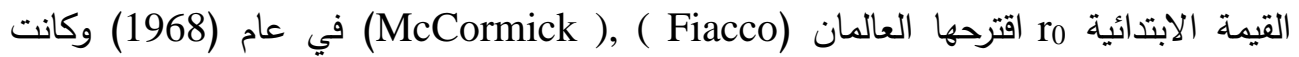

$$
r_{0}=\frac{\nabla f(x)^{T} \nabla B(x)}{\nabla B(x)^{T} \nabla B(x)}
$$

وتم ايجاد عدة بدائل لقيمة ro راجع (Al-Bayati and Hamed , 2000) و Al-Bayati and ) Hamed , 1999).

في هذا البحث تم استخدام خوارزمية BFGS المتوازية والمشروطة المقترحة من قبل (Al-Bayati and Aref , 2001)

$$
\text { 2. خوارزميات التدرج المترافق المشروط مسبقا": }
$$

\section{Preconditioned Conjugate Gradient Methods (PCG)}

خوارزميات أشباه نيوتن-Quasi-Newton (QN) والتدرج المترافـق

Conjugate Gradient (CG) خوارزمية QN أسرع (وتتطلب حسابات اقل لقيمة الدالة المعينة) من خوارزميات التدرج المترافق كذلك خوارزميات QN تكون متتابعة من مصفوفات متتاظرة، مربعة وموجبة قطعاً ، خوارزميات

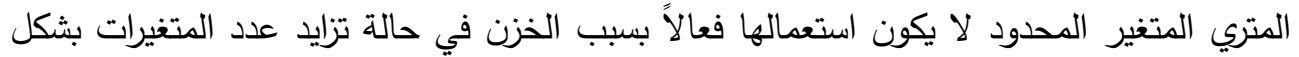

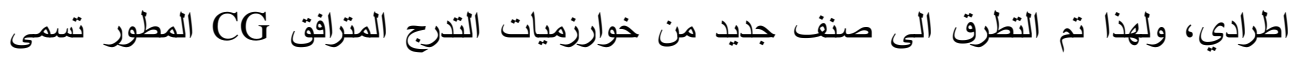
خوارزميات التدرج المترافق المشروط (PCG). فكرة الاشتراط هي لتحويل المسألة، إذ ان مصفوفة well - للمسألة المتحولة تمتلك القيم المميزة المتجمعة وتكون حسنة الشرط لفئل conditioned

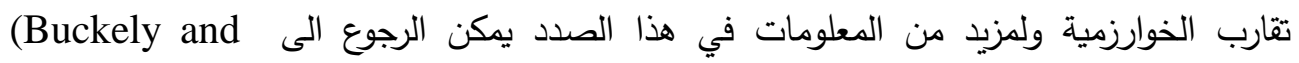

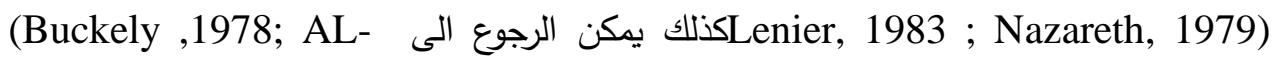


في خوارزميات Bayati and Mohammed Ali, 2002) المترافق يتولد بالاعتماد على تحويلات المصفوفة.

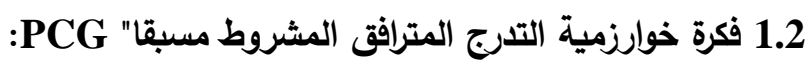

\section{The Idea of the PCG Algorithm:}

Axelsson, ) خوارزمية CG PCG القياسية والتي اقترحها في البداية PG 1974) واستعملت في حل انظمة المعادلات الخطية، فكرة الاشتراط امتدت مباشرة لحل المسائل غير الخطية.

هذا النوع من خوارزميات التدرج المترافق يكون ملائماً للمسائل التي تكون متغيراتها كبيرة.

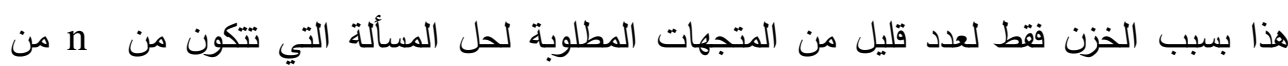

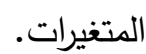

إن خوارزمية CG الأصلية ليست فعالة دائماً لكن الاشتراط يستعمل المصفوفة المناسبة

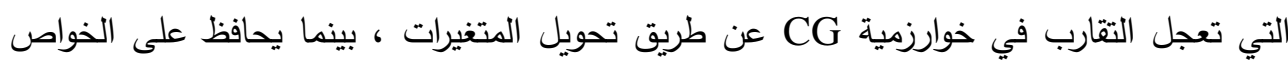

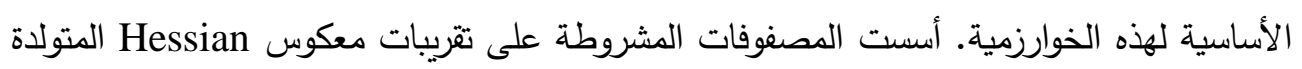

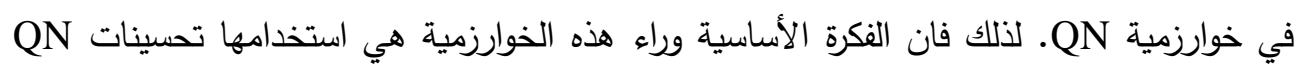

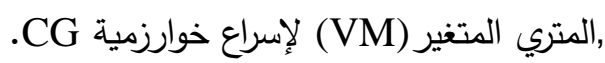

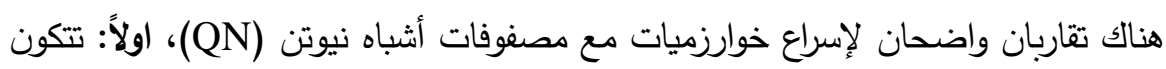

من تطبيق خوارزميات CG مع المتري المتغير المطور وباستعمال صيغة QN. هذه هندات الخوارزمية

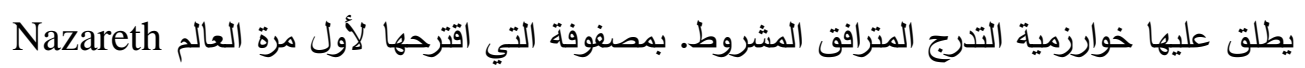
في (1979) ومن ثم طورها آخرون أمثال(Al-Bayati and Sharef, 2001).

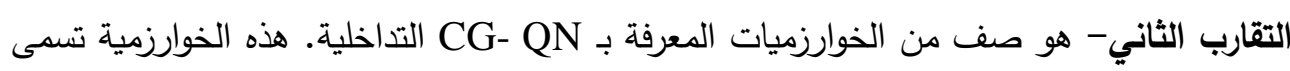
(Interleaved) حيث اقترحها لأول مرة العالم الكندي (Buckely,1987) المتداخلة CG-QN وطورها (Shanno, 1981) و (Nazareth and Nocedal, 1982) و ( B (Suckely and ) و (Liu and Nocedal, 1988) و (Lenir, 1983 - (Al-Bayati and Mohammed Ali, 2002)

\section{Preconditioning with Quasi - Newton Approximations}

2.2 تقرببات أشباه نيوتن بوجود الاشتراط المسبق:

الاشتراط في خوارزميات التدرج المترافق يعني تحسين أدائها ـ وتتطلب فعالية الاشتراط

معلومات عن المشتقة الثانية التي تكون غير متوافرة أحيانا ويمكن جمعها في طريقة خوارزمية

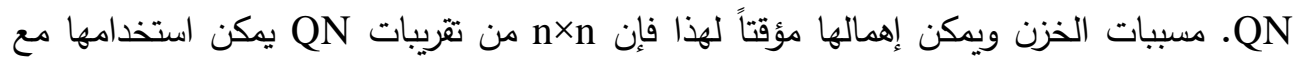


بعض الشروط الموضوعة عن خط البحث والدوال غير الخطية ، المصفوفات $H_{k}$ لتقريب معكوس Hessain مكان للحصول على التوقف التربيعي مع خوارزمية التدرج المترافق. .(Alهذا بالنسبة للنماذج التربيعية, اما في النماذج غير التربيعية فيمكن الرجوع الى فئى Bayati and Al-Assady, 1997)

2.3 تقريبات QN محدودة الذاكرة :

\section{Limited - Memory Quasi - Newton Approximations}

أثبت تحديد الذاكرة لتقريبات QN معنى اخر للاشتراط مع تقريبات QN ، ويمكن اعتبار

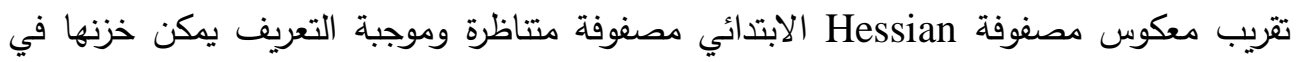
الذاكرة المتوافرة حتى وان ضربت في متجه سعته O(n) من العمليات، عادة ما تكون مصفوفة

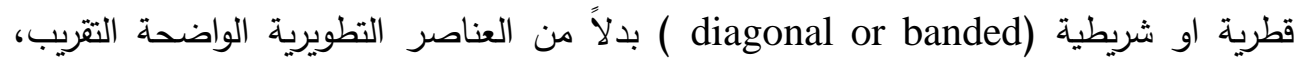
ويكون التطوير تماماً بواسطة خزن المتجهات مع المعلمات التي تعرف بتطوير (Shanno, 1982)

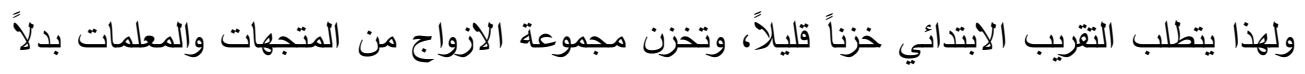

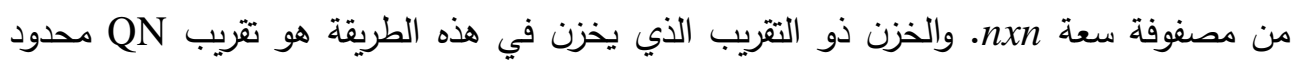

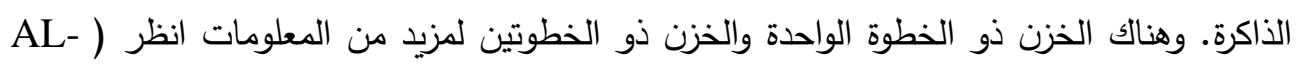
.(Bayati and Ahmed, 1997

\section{Improved BFGS Algorithm}

3. خوارزمية BFGS

sk في خوارزميات المتري المتغير المشروط المعروفة نبدأ من نقطة اختيارية لاتجاه البحث

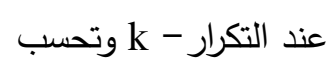

$$
s^{k}=-H_{k} \Delta f\left(x^{k}\right)
$$

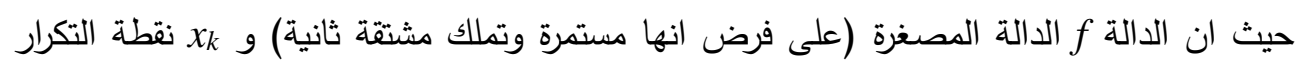
المتداولة ، و Hessian عند $H_{k}$ التقريب الى معكوس مصفة التكرار التالي نحصل عليها من :

$$
x^{k+1}=x^{k}+\lambda_{k} s^{k}
$$

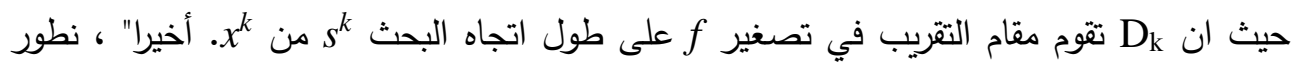
بواسطة إضافتها الى المصفوفة D $H_{k}$ $H_{k+1}=H_{k}+D_{k}$

$$
\sigma^{k}=x^{k+1}-x^{k}
$$




$$
y^{k}=g\left(x^{k+1}\right)-g\left(x^{k}\right)
$$

$$
\text { حيث }
$$

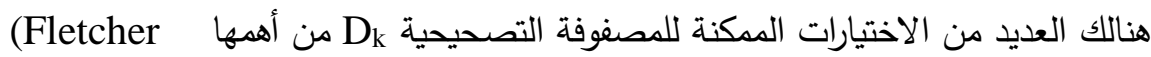
and Powell, 1963 ; Broyden, 1970 ; Davidon , 1959 ; Al-Bayati, 1991 ).

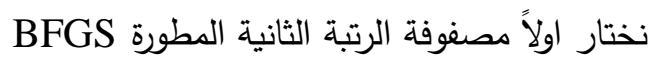

$$
D_{k}=\frac{H_{k} y^{k}\left(y^{k}\right)^{T} H_{k}}{\left(y^{k}\right)^{T} H_{k} y^{k}}+\frac{\sigma^{k}\left(\sigma^{k}\right)^{T}}{\left(\sigma^{k}\right)^{T} y^{k}}+w^{k}\left(w^{k}\right)^{T}
$$$$
\text { حيث ان D }
$$

$$
w^{k}=\left(\left(y^{k}\right)^{T} H_{k} y^{k}\right)^{\frac{1}{2}}\left(\frac{\sigma^{k}}{\left(\sigma^{k}\right)^{T} y^{k}}-\frac{H_{k}\left(y^{k}\right)^{T}}{\left(y^{k}\right)^{T} H_{k} y^{k}}\right)
$$

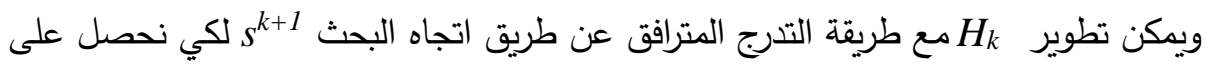
$s^{k+1}=-H_{k+1} g^{k+1}+\frac{\left(g^{k+1}\right)^{T} H_{k+1} g^{k+1}}{\left(g^{k}\right)^{T} g^{K}} s^{k}$

حيث ان لكزيد من المعلومات عن خوارزمية BFGS انظر (Al-Bayati and Ahmed, 1996). 4. خطوات خوارزمية BFGS المتوازية والمشروطة مسبقا":

\section{(Al-Bayati and Aref, 2001)}

\section{خوارزمية (1)}

الخطوة الاولى:- لتكن x نقطة اختيارية مناسبة, ${ }^{0}$ مصفوفة ابتدائية وتكون عادة مصفوفة الوحدة

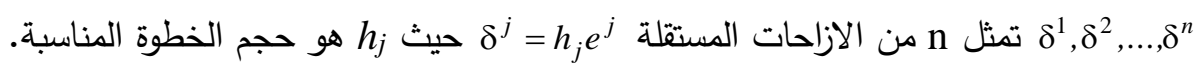

$$
\begin{aligned}
& s^{0}=-H_{1} g\left(x^{0}\right)-{ }^{-} \text {: الخطوة الثانية }
\end{aligned}
$$

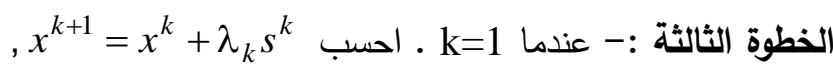

$$
\begin{aligned}
& \underset{\lambda_{k}}{\arg \min } f\left(x^{k}+\lambda_{k} s^{k}\right)=E L S \text { حيث } \lambda_{k} \text { تحسب : } \\
& \text { الخطوة الرابعة :-راجع إذا كان ع >||| } \\
& \text { الخطوة الخامسة :- احسب في التوازي ( }
\end{aligned}
$$

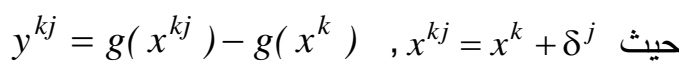

$$
\begin{aligned}
& \text { واحسب }
\end{aligned}
$$




$$
\begin{aligned}
& \gamma^{k j}=\frac{\delta^{j}}{\left(y^{k j}\right)^{T} \delta^{j}}-\frac{H_{k} y^{k j}}{\left(y^{k j}\right)^{T} H_{k} y^{k j}} \\
& \text { وتحسن أن من خلال } \\
& H_{k+1}=H_{k}-\frac{H_{k} y^{k j}\left(y^{k j}\right)^{T} H_{k}}{\left(y^{k j}\right)^{T} H_{k} y^{k j}}+\frac{\delta^{k}\left(\delta^{k}\right)^{T}}{\left(\delta^{k}\right)^{T} y^{k j}}+\left[\left(y^{k j}\right)^{T} H_{k} y^{k j}\right]^{\frac{1}{2}}\left(\gamma^{k j}\right)\left(\gamma^{k j}\right)^{T} \\
& \text { الخطوة السادسة :احسب الاتجاه المطور } \\
& s^{k+1}=-H_{k+1} g^{k+1}+\frac{\left(g^{k+1}\right)^{T} H_{k+1} g^{k+1}}{\left(g^{k}\right)^{T} g^{K}} s^{k}
\end{aligned}
$$

الخطوة السابعة : أجريت عملية الاسترجاع من البداية بوضع و=1 او او استرجاع Powell او حيث ان الثرط الأخير يضمن $H_{k}\left(g^{k+1}\right)^{T} H_{k} g^{k+1}>0$ لمزيد من المعلومات انظر (Al-Bayati and Aref, 2001) حول موضوع التوازي .

5. الخوارزمية المقترحة الى BFGS المتوازية والمشروطة المقيدة :

\section{خوارزمية (2)}

الخطوة الأولى والثانية / كما في خوارزمية (1) الخطوة الثالثة : احسب

$$
\phi(x, r)=f(x)+\sum_{k=1}^{m} r_{k} B\left\{c_{i}(x)\right\}
$$

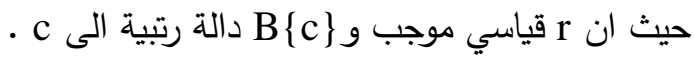
الخطوة الرابعة :- احسب $\arg \min f\left(x^{k}+\lambda_{k} s^{k}\right)=E L S$

الخطوة الخامسة :-افحص إذا كان ع >||( والا اذهب الى الخطوة السادسة.

$$
\begin{aligned}
& \text { الخطوة السادسة :- احسب في التوازي ( } \\
& y^{k j}=g\left(x^{k j}\right)-g\left(x^{k}\right) \quad, x^{k j}=x^{k}+\delta^{j} \quad \text { حين } \\
& \text { واحسب } \\
& \gamma^{k j}=\frac{\delta^{j}}{\left(y^{k j}\right)^{T} \delta^{j}}-\frac{H_{k} y^{k j}}{\left(y^{k j}\right)^{T} H_{k} y^{k j}}
\end{aligned}
$$




$$
\begin{aligned}
& H_{k+1}=H_{k}-\frac{H_{k} y^{k j}\left(y^{k j}\right)^{T} H_{k}}{\left(y^{k j}\right)^{T} H_{k} y^{k j}}+\frac{\delta^{k}\left(\delta^{k}\right)^{T}}{\left(\delta^{k}\right)^{T} y^{k j}}+\left[\left(y^{k j}\right)^{T} H_{k} y^{k j}\right]^{\frac{1}{2}}\left(\gamma^{k j}\right)\left(\gamma^{k j}\right)^{T} \\
& \text { الخطوة السابعة :احسب الاتجاه: } \\
& s^{k+1}=-H_{k+1} g^{k+1}+\frac{\left(g^{k+1}\right)^{T} H_{k+1} g^{k+1}}{\left(g^{k}\right)^{T} g^{K}} s^{k} \\
& \text { الخطوة الثامنة : افحص التقارب }
\end{aligned}
$$

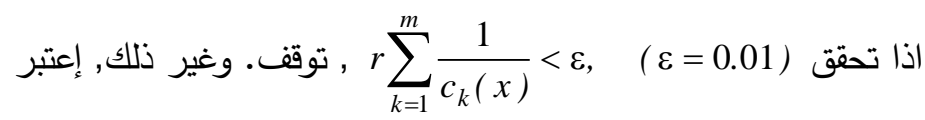

$$
\begin{aligned}
& x=x^{*}, \quad r_{k+1}=\frac{r_{k}}{10} \\
& \text { بوضع k=k+1 , واذهب الى الخطوة الاولى. }
\end{aligned}
$$

\section{Results and Conclusions}

5. - 5 النتائج والحسابات:

تضمنت إختبارات المقارنة (5) دوال اختبار مقيدة معروفة بابعاد تتراوح بين 3 بn $1 \leq$ و و

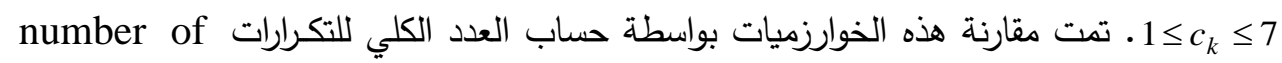
number of function evaluation (NOF) مع العدد الكلي لقيم الدوالiteration (NOI)

$$
\begin{aligned}
& \text { - ث اخذ مقياس التوقف ع ع>||| } \\
& \text { هناك نوعان من الخوارزميات التي تم اختبارها: } \\
& \text { (1) خوارزمية SUMT) } \\
& \text { (2) (1) (1) (الخوارزمية المقترحة. }
\end{aligned}
$$

الجدول رقم (1) تضمن مقارنة بين الخوارزميتين (1), (2). حيث يبين هذا الجدول ان نتائج الخوارزمية المقترحة أفضل من نتائج الخوارزمية الأصلية. إن فكرة الاشتراط المتوازي تم استخدامها هنا لاول مرة في الامثلية المقيدة حيث حصلنا على نلى نتائج مشجعة في التجارب العملية.

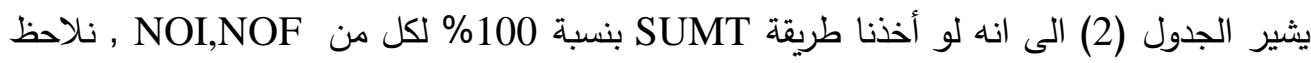

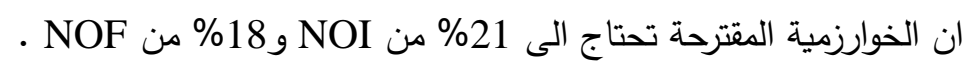


Table (1)

SUMT مقارنة الخوارزمية الجديدة مع خوارزمية

\begin{tabular}{|c|c|c|}
\hline Test function & $\begin{array}{c}\text { SUMT algorithm } \\
\text { NOI (NOF) }\end{array}$ & $\begin{array}{c}\text { New algorithm } \\
\text { NOI (NOF) }\end{array}$ \\
\hline 1. & $32(129)$ & $26(106)$ \\
\hline 2. & $24(81)$ & $21(73)$ \\
\hline 3. & $37(137)$ & $27(99)$ \\
\hline 4. & $13(50)$ & $10(42)$ \\
\hline 5. & $39(122)$ & $31(100)$ \\
\hline Total & $145(519)$ & $115(420)$ \\
\hline
\end{tabular}

Table (2)

\begin{tabular}{|c|c|c|}
\hline & SUMT Algorithm & New Algorithm \\
\hline NOI & $100 \%$ & 79.3 \\
\hline NOF & $100 \%$ & 82.3 \\
\hline
\end{tabular}

\section{Appendix}

\section{Test functions}

(1) $\min f(x)=\left(x_{1}-2\right)^{2}+\left(x_{2}-1\right)^{2}$ s.p ( 2,7)

s.t

$$
x_{1}-2 x_{2}=-1
$$

$\frac{-x_{1}^{2}}{4}+x_{2}^{2}+1 \geq 0$

(2) $\min f(x)=-x_{1}^{2} x_{2}$

s.t

$$
\begin{aligned}
& x_{1} x_{2}+\left(x_{1}^{2}\right) / 2=6 \\
& x_{1}+x_{2} \geq 0
\end{aligned}
$$

(3) $\min f(x)=x_{1}^{2}+x_{2}^{2}$ S.p $(0.9,2)$

s.t.

$x_{1}+2 x_{2}=4$

$x_{1}^{2}+x_{2}^{2} \leq 5$

$x_{i} \geq 0$

(4) $\min f(x)=\left(x_{1}-x_{2}\right)^{2}+\left(x_{2}-2 x_{2}\right)^{2}$ S.p $(\mathbf{0 , 1})$

s.t.

$x_{1}^{2}-x_{2} \leq 0$

(5) $\min f(x)=x_{1} x_{4}\left(x_{1}+x_{2}+x_{3}\right)+x_{3}$ $x_{0}=(4,3,3,3)$

s.t.

$x_{1}^{2}+x_{2}^{2}+x_{3}^{2}+x_{4}^{2}=40$

$x_{1} x_{2} x_{3} \geq 25$

$5 \geq x_{i} \geq 1$ 
المصادر

[1] Al-Bayati A.Y. and Aref, S. (2001), “Modified parallel Self-Scaling variable metric algorithms”, M.Sc. Thesis, Dep. Of Math., University of Mosul.

[2] Al-Bayati, A.Y. and Hamed, E.T. (1998), "New self-scaling sequential algorithm for the minimization of constrained nonlinear function", J. of Dirasat, Jordan, Vol (25), PP. 339-351.

[3] Al-Bayati, A.Y. and Hamed, E.T. (2000), "New Parameter for the inverse Barrier method in constrained nonlinear optimization" J. of Educ. And Sci. Mosul, Vol. (44), PP. 93-110.

[4] Al-Bayati, A.Y. and Shareef, S.G. (2001), “ A modification PCGmethod for non-linear optimization”, Al-Rafidain J.of Sci. Mosul, Iraq.

[5] Al-Bayati A.Y. and Al-Assady N.H (1997), “ PCG-Methods for nonlinear optimization J. of Mo'tah, Jordan, Vol (12).

[6] Al-Bayati, A.Y. and Ahmed, H.I. (1997), “ Investigation on double update variable storage CG-method", J. of Al-Yarmouk, Jordan, Vol (6), pp. 23-41.

[7] Al-Bayati, A.Y. and Ahmed, H.I. (1996), “ Investigation on single update memoryless CG-method”, Qatar University Sci. J. Vol (16), pp. 183-192.

[8] Al-Bayati, A.Y. and Mahammed Ali, M.M. (2002), "Multi-Step hybrid CG-algorithm for Unconstrained optimization, Al-Rafidain J. of Sci., Mosul, Iraq, Vol. (13), pp. 94-101.

[9] Al-Bayati, A.Y. (1991), “ A New Family of Self-Scaling Variable Metric Algorithms for Unconstrained Optimization", J of Education on Science, Mosul University.

[10] Axelsson, O. (1974), “ On Preconditioning and Convergence Acceleration in Sparse Matrix Problems", CERN data Hunding Division Report, pp. 74-100.

[11] Buckley, A.G. and Lenir, A. (1983), "Quasi-Like Variable Storage Conjugate Gradients", Math. Prog. 27. Pp. 155-175.

[12] Buckley, A.G. (1978a), “ A Combined CG-QN Minimum Algorithm, Math. Prog. 15, pp. 200-210. 
[13] Broyden, C. G. (1970), "The Convergence of a Class of DoubleRank Minimization Algorithm", J. Inst. Apl. 6. pp. 66-90 and 22223.

[14] Davidon, W.C. (1959), "Variable Metric Method for Minimization". AERC and D. Report A. NI-5990. Argonne II.

[15] Fletcher, R. and Powell, M.J.D. (1963), “A Rapidly Convergent Descent Method for Minimization”, Comp. J. 6, pp. 163-168.

[16] Fiacco, A.V. and McCormik, G.P. (1968), “ Non-Linear Programming, Sequential Unconstrained Minimization Technique”. Wiley, New York.

[17] Liu, D.C. and Nocedal, J. (1988), "On the Limited Memory BFGS Method for Large-Scale Optimization:, Rep. NAM 03, Dept. of Electrical Engineering and Computer Science, North Wastrel University, Envonstor II.

[18] Nazareth, L. and Nocedal, J. (1982). " Conjugate Direction Methods with Variable Storage”, Math. Prog. 23, pp. 341-348.

[19] Nazareth, L. (1979), " A Relationship Between the BFGS and Conjugate Algorithms and Implications for New Algorithms", SIAM J. Number, Anal. 16. Pp. 794-800.

[20] Shanno, D.F. (1981), On PCG-Methods" In: Non-Linear Programming 4. Eds. O. L. Mangasarian, R.R. Mayer and S.M. Robinson, Academic Press, New York, of QN Methods for function Minimization. Math. Comp. 24, pp. 647-656. 